\title{
Long non-coding RNA HOTAIR knockdown alleviates lipopolysaccharide-induced acute respiratory distress syndrome and the associated inflammatory response by modulating the microRNA-30a-5p/PDE7A axis
}

\author{
HONGRONG WANG* ${ }^{*}$, SHASHA SONG ${ }^{*}$ and XIANYU MU \\ Department of Emergency, Yantai Yuhuangding Hospital, Yantai, Shandong 264000, P.R. China
}

Received August 20, 2020; Accepted May 7, 2021

DOI: 10.3892/etm.2021.10594

\begin{abstract}
Acute respiratory distress syndrome (ARDS) is a severe pulmonary disease, which can be modulated by certain long non-coding (lnc)RNAs. The present study aimed to investigate the regulatory mechanism of IncRNA HOTAIR in ARDS and the inflammatory response induced by lipopolysaccharide (LPS). The mRNA expression levels of HOTAIR, microRNA ( $m i R)-30 a-5 p$ and $P D E 7 A$ were determined using reverse transcription-quantitative PCR, while a MTT assay was used to assess the viability of the MLE-12 cells and ELISA was used to determine the concentration of different inflammatory factors [tumor necrosis factor (TNF)- $\alpha$, IL-1 $\beta$ and IL-6]. The interactions between miR-30a-5p and HOTAIR/PDE7A were predicted using TargetScan and StarBase databases and verified using a dual-luciferase reporter assay. The protein expression levels of PDE7A were determined using western blot analysis. Mouse models of LPS-induced ARDS were established to investigate the suppressive effect of HOTAIR knockdown on ARDS in vivo. IncRNA HOTAIR was increased in LPS-treated MLE-12 cells and in a ARDS mouse model. HOTAIR knockdown decreased the concentration of TNF- $\alpha$, IL- $1 \beta$ and IL-6, and increased cell viability in vitro. miR-30a-5p upregulation decreased TNF- $\alpha$, IL-1 $\beta$ and IL-6 concentrations, and increased cell viability in vitro. HOTAIR targeted miR-30a-5p and miR-30a-5p targeted PDE7A. miR-30a-5p downregulation and PDE7A upregulation reversed the suppressive effect of HOTAIR knockdown on the concentrations of TNF- $\alpha$, IL-1 $\beta$ and IL-6, and the positive effect of HOTAIR knockdown on cell viability
\end{abstract}

Correspondence to: Dr Xianyu Mu, Department of Emergency, Yantai Yuhuangding Hospital, 20 Yuhuangding East Road, Zhifu, Yantai, Shandong 264000, P.R. China

E-mail: muxianyu270@163.com

*Contributed equally

Key words: acute respiratory distress syndrome, inflammatory response, HOTAIR, miR-30a-5p, PDE7A in vitro. HOTAIR knockdown also attenuated ARDS and the inflammatory response induced by LPS in vivo. The suppression of HOTAIR alleviated ARDS and the inflammatory response induced by LPS by modulating the $m i R-30 a-5 p / P D E 7 A$ axis. These results provide a potential therapeutic strategy for ARDS.

\section{Introduction}

Acute respiratory distress syndrome (ARDS) is the extreme manifestation of acute lung injury (ALI) (1). In 2016, estimates of the incidence of ARDS in high- and middle-income countries varied between 10.1 and 86.2 per 100,000 individuals per year in the general population (2). As a pulmonary disease that occurs in response to stimuli targeting the alveolar-capillary membrane, ALI/ARDS can lead to increased vascular permeability and subsequently, pulmonary oedema (3). Lipopolysaccharide (LPS) is considered to be a major active factor during the inflammatory response $(4,5)$. LPS-induced inflammation has been reported to play a critical role in the pathogenesis of ALI (6). Consequently, further molecular research on the potential mechanisms of LPS-induced inflammatory factor release in ALI/ARDS and the development of new therapeutic targets for ARDS are urgently required.

There is increasing evidence that long non-coding (lnc) RNAs have been associated with regulating pulmonary diseases, including ALI/ARDS (7-9). Dai et al (7) showed that MALAT1 overexpression increased the concentration of LPS-induced inflammatory factors in mouse alveolar cells; therefore, accelerating the progression of LPS-induced ALI. Wang et al (8) revealed that THRIL mRNA expression level was positively correlated with IL- $1 \beta$ and TNF- $\alpha$ concentration in the tissues of patients with ARDS. Zhou et al (9) found that NEAT1 expression was markedly elevated in a mouse model of ALI and NEAT1 knockdown inhibited the release of inflammatory factors from mouse alveolar epithelial cells. Notably, HOTAIR has been associated with regulating the LPS-induced inflammatory response in different aspects, such as LPS-induced inflammatory injury in mouse chondrogenic cells (10), and in the LPS-induced inflammatory response in mice macrophages (11) and in cardiomyocytes from mice 
with sepsis (12). However, the detailed mechanism of HOTAIR in the LPS-induced inflammatory response in ALI/ARDS remains unknown.

It is widely known that microRNAs (miRNA/miR) have been associated with LPS-induced ALI and the release of inflammatory mediators (13-15). Li et al (13) revealed that the overexpression of $m i R-150$ decreased the concentration of IL-6, IL-1 $\beta$ and tumor necrosis factor (TNF)- $\alpha$ in mice with LPS-induced ALI. Cheng et al (14) demonstrated that $m i R-424$ upregulation inhibited the release of inflammatory factors from LPS-induced alveolar epithelial cells. He et al (15) found that lung injury and the inflammatory response induced by LPS were attenuated by $m i R-146 b$ overexpression; therefore, ameliorating LPS-induced ALI. Notably, $m i R-30 a-5 p$ has been shown to have a protective role in LPS-induced ALI in children (16). In addition, lncRNAs can act as competing endogenous RNAs or sponges of miRNAs. HOTAIR has been reported to regulate numerous miRNAs in several types of cancer, such as $m i R-138-5 p$ in ovarian cancer (17), $m i R-1277-5 p$ in gastric cancer (18), $m i R-449 b-5 p$ (19) or $m i R-601$ (20) in breast cancer and $m i R-203$ in lung cancer (21). However, the mechanisms whereby lncRNA HOTAIR regulates $m i R-30 a-5 p$ in ARDS/ALI have not yet been reported.

In the present study the effects of IncRNA HOTAIR knockdown on the concentrations of inflammatory factors in MLE-12 cells, the regulatory mechanism of how HOTAIR regulates the $m i R-30 a-5 p / P D E 7 A$ axis in LPS-induced MLE-12 cells and the effect of HOTAIR knockdown on LPS-induced ARDS in vivo were investigated. The results are aimed to provide a promising therapeutic target for ARDS.

\section{Materials and methods}

Cell culture, grouping and transfection. The murine alveolar epithelial cell line, MLE-12, was purchased from Baiye Biotech, Ltd. The MLE-12 cells were cultured at $37^{\circ} \mathrm{C}$ in a humidified incubator with $5 \% \mathrm{CO}_{2}$, in DMEM (Invitrogen; Thermo Fisher Scientific, Inc.), supplemented with $10 \%$ fetal bovine serum (Gibco; Thermo Fisher Scientific, Inc.) and 0.1\% penicillin/streptomycin.

A short hairpin (sh)RNA-non-targeting negative control (sh-NC) and shRNA targeting HOTAIR (sh-HOTAIR) were purchased from Sangon Biotech, Co., Ltd. A PDE7A-overexpression plasmid (pcDNA-PDE7A) and its NC (pcDNA-NC), $m i R-30 a-5 p$ mimics/miR-NC and $m i R-30 a-5 p$ inhibitor/inhibitor NC were all purchased from Guangzhou RiboBio Co., Ltd. The aforementioned molecules (all at $20 \mathrm{nM}$ ) were transfected into the MLE-12 cells using Lipofectamine ${ }^{\circledR} 3000$ (Invitrogen; Thermo Fisher Scientific, Inc.) at $37^{\circ} \mathrm{C}$ for $48 \mathrm{~h}$. Subsequently, the transfected cells were induced with LPS (1 $\mu \mathrm{g} / \mathrm{ml}$; Sigma-Aldrich; Merck KGaA). MLE-12 cells without any treatments were served as the controls. The next day, the MLE-12 cells were harvested for the following experiments.

Reverse transcription-quantitative PCR (RT-qPCR). Total RNA was extracted from the mouse cells or lung tissues using TRIzol $^{\circledR}$ (Invitrogen; Thermo Fisher Scientific, Inc.). Total RNA (500 ng) was reverse transcribed into cDNA at $42^{\circ} \mathrm{C}$ for
45 min using a First-Strand cDNA Synthesis kit (APeXBIO Technology LLC) and qPCR was performed with SYBR Green FAST MasterMix kit (Qiagen $\mathrm{GmbH}$ ), according to the manufacturer's instructions. The following thermocycling conditions were used for qPCR: Initial denaturation at $94^{\circ} \mathrm{C}$ for $10 \mathrm{~min}$, followed by 40 cycles at $94^{\circ} \mathrm{C}$ for $10 \mathrm{sec}, 60^{\circ} \mathrm{C}$ for $20 \mathrm{sec}$ and $72^{\circ} \mathrm{C}$ for $1 \mathrm{~min}$. GADPH and $U 6$ were used as the internal references. The respective sequences of primers were as follows: HOTAIR forward, 5'-GGTAGAAAAAGCAAC CACGAAGC-3' and reverse, 5'-ACATAAACCTCTGTCTGT GAGTGCC-3'; miR-30a-5p forward, 5'-AACGAGACGACG ACAGAC-3' and reverse, 5'-TGTAAACATCCTCGACTG GAAG-3'; PDE7A forward, 5'-GGAAATAGTCTAGTAAGC TTAACC-3' and reverse, 5'-GGCAGATGTGAGAATAAG CCTG-3'; GAPDH forward, 5'-TCCGCCCCTTCCGCTGA TG-3' and reverse, 5'-CACGGAAGGCCATGCCAGTGA-3'; U6 forward, 5'-CTCGCTTCGGCAGCACA-3' and reverse 5'-AACGCTTCAGAATTTGCGT-3'. Gene expression was quantified using the $2^{-\Delta \Delta \mathrm{Cq}}$ method (22).

MTT assay. The viability of the MLE-12 cells was determined using a MTT assay. Transfected and LPS-treated MLE-12 cells were seeded into a 96 -well plate, at $2 \times 10^{5}$ cells per well and incubated at $37^{\circ} \mathrm{C}$. After incubation for $24 \mathrm{~h}, 20 \mu \mathrm{l}$ MTT (Shanghai GeneChem, Co., Ltd.) was added to each well. After incubation for $2 \mathrm{~h}$ at $37^{\circ} \mathrm{C}$, cell viability (optical density at $570 \mathrm{~nm}$ ) was analyzed using a Multiskan Spectrum microplate reader (Thermo Fisher Scientific, Inc.).

ELISA. The concentration of the inflammatory factors [TNF- $\alpha$ (cat. no. 70-EK182HS-96), IL-1 $\beta$ (cat. no. 70-EK101BHS-96) and IL-6 (cat. no. 70-EK106/2-24)] in the MLE-12 cells or mouse bronchoalveolar lavage fluid (BALF) were measured using specific ELISA kits (Multisciences Biotech Co., Ltd.), according to the manufacturer's protocol. A Multiskan Spectrum microplate reader (Thermo Fisher Scientific, Inc.) was used to determine the absorbance at $450 \mathrm{~nm}$.

Target prediction. The miRNA targets of HOTAIR were predicted using StarBase database (http://starbase.sysu.edu.cn/). In addition, the mRNA targets of $m i R-30 a-5 p$ were predicted using TargetScan database (http://www.targetscan.org).

Dual luciferase reporter (DLR) assay. The 3'-untranslated region sequences containing the HOTAIR binding site were cloned into the pGL3 vector (Promega Corporation) to establish the wild-type (WT) vector. The Phusion Site-Directed Mutagenesis kit (Thermo Fisher Scientific, Inc.) was used to construct the mutant vector. The WT or mutant vectors and the miR-146a mimics or miR-NC were co-transfected into the MLE-12 cells using Lipofectamine 3000 (Invitrogen; Thermo Fisher Scientific, Inc.) at $37^{\circ} \mathrm{C}$ for $48 \mathrm{~h}$. A DLR Assay System (Promega Corporation) was used to determine relative luciferase activity. The activity of firefly luciferase was normalized to the activity of Renilla luciferase.

Western blot analysis. RIPA buffer (Beyotime Institute of Biotechnology), containing protease inhibitors was used to extract total protein from the MLE-12 cells. Protein concentration was then determined using a BCA Protein Assay kit 
(Abcam). A total of $50 \mu \mathrm{g}$ protein/lane was separated using $10 \%$ SDS-PAGE and the resolved proteins were transferred onto PVDF membranes. The membranes were blocked with $5 \%$ bovine serum albumin (Thermo Fisher Scientific, Inc.) for $2 \mathrm{~h}$ at room temperature. Following which, the membranes were incubated overnight at $4{ }^{\circ} \mathrm{C}$ with the primary antibodies against PDE7A (1:1,000; cat. no. ab14616; Abcam) and tubulin (1:1,000; cat. no. ab6160; Abcam). Subsequently, the membranes were washed 3 times with TBS-Tween-20 $(0.05 \%)$, then incubated with an HRP-conjugated anti-rabbit IgG secondary antibody (1:5,000; cat. no. ab6721; Abcam) and an HRP-conjugated anti-rat IgG secondary antibody (1:5,000; cat. no ab6734; Abcam), respectively, at room temperature for $1 \mathrm{~h}$. Tubulin was used as the internal reference. An enhanced chemiluminescence detection kit (Thermo Fisher Scientific, Inc.) was used to detect the bands, which were then quantified using Gel-Pro Analyzer software (v4.0; Media Cybernetics, Inc.).

Construction of an ARDS mouse model. A total of 20 female BALB/c nude mice (4-5 weeks; weighing $20 \pm 2 \mathrm{~g}$ ) were purchased from Cavens Lab. All mice were housed under controlled conditions $\left(25^{\circ} \mathrm{C} ; 50 \%\right.$ humidity; $12 \mathrm{~h} \mathrm{light/dark}$ cycle) and had free access to food and water. The mouse model of ARDS was constructed by intratracheal instillation of $2 \mathrm{mg} / \mathrm{kg}$ LPS. The mice were randomly divided into sham, ARDS, ARDS + sh-NC and ARDS + sh-HOTAIR groups $(n=5)$. After LPS treatment for $48 \mathrm{~h}$, the mice in the ARDS + sh-HOTAIR group were treated with sh-HOTAIR lentivirus $\left(2 \times 10^{7}\right.$ transducing units $/ \mathrm{ml}$ by intravenous injection), while those in the ARDS + sh-NC group were intravenously injected with an equal quantity of sh-NC lentivirus. Simultaneously, the mice in the sham group were intravenously injected with an equal volume of saline. The next day, the mice were anesthetized with sodium pentobarbital $(50 \mathrm{mg} / \mathrm{kg})$ and euthanized via cervical dislocation. Subsequently, the BALF samples were collected and centrifuged, and the resulting supernatants were used to measure the concentration of TNF- $\alpha$, IL-6 and IL-1 $\beta$.

Histopathological examination using $H \& E$ staining. Fresh mouse lung samples were resected, then fixed with $4 \%$ paraformaldehyde at $37^{\circ} \mathrm{C}$ for 1 day. The samples were then embedded in paraffin and cut into $6-\mu \mathrm{m}$ thick sections. The sections were immediately stained with $\mathrm{H} \& \mathrm{E}$ for $15 \mathrm{~min}$ at room temperature, then observed using a light microscope (magnification, $\mathrm{x} 200$ ). The injury score was calculated based on a previous study (23).

Lung function assay. After sacrifice of the mice, arterial blood samples $(5 \mathrm{ml})$ were collected from mouse carotid arteries. An automatic blood gas analyzer (Radiometer Medical ApS) was used to determine the partial pressure of oxygen $\left(\mathrm{PaO}_{2}\right)$ and carbon dioxide $\left(\mathrm{PaCO}_{2}\right)$. The wet weight of the fresh lung tissues was then determined. The tissues were dried at $60^{\circ} \mathrm{C}$ for $72 \mathrm{~h}$ and the dry weight was then determined. The wet/dry weight ratio of the lung samples was used as a measure of tissue oedema.

Statistical analysis. SPSS software (v20.0; IBM, Corp.) was used to perform statistical analyses. The data are presented as the mean \pm standard deviation. A Student's t-test (unpaired) was used to assess the differences between two groups, while one-way ANOVA was used to evaluate the differences among multiple groups, followed by a Tukey's multiple comparisons test. $\mathrm{P}<0.05$ was considered to indicate a statistically significant difference. All experiments were performed in independently 3 times in triplicate.

\section{Results}

IncRNA HOTAIR knockdown decreases the concentration of the inflammatory factors in LPS-induced MLE-12 cells. HOTAIR mRNA expression level was upregulated in the MLE-12 cells in the LPS group compared with that in the control group $(\mathrm{P}<0.01$; Fig. 1A). RT-qPCR was then used to determine the transfection efficiency of sh-HOTAIR and sh-NC in the MLE-12 cells and the results illustrated that HOTAIR expression was significantly decreased in the sh-HOTAIR group compared with that in the sh-NC group $(\mathrm{P}<0.01$; Fig. 1B). A MTT assay revealed that, compared with that in the control cells, treatment with LPS significantly decreased cell viability. Cell viability in the sh-HOTAIR + LPS group was also significantly increased compared with that in the sh-NC + LPS group $(\mathrm{P}<0.01$; Fig. 1C). ELISA results showed that the concentration of IL-6, IL-1 $\beta$ and TNF- $\alpha$ were all upregulated in the MLE-12 cells in the LPS group compared with that in the control group. However, transfection with sh-HOTAIR significantly decreased the concentration of the inflammatory factors in the MLE-12 cells treated with LPS compared with that in cells transfected with sh-NC $(\mathrm{P}<0.01$; Fig. 1D-F).

lncRNA HOTAIR targets miR-30a-5p. The potential binding sites between HOTAIR and $m i R-30 a-5 p$ were predicted using StarBase database (Fig. 2A). Among the 46 miRNA targets predicted, miR-30a-5p was selected for further analysis as it has been associated with ALI (16) and its unknown regulatory association with HOTAIR. The mRNA expression levels of $m i R-30 a-5 p$ following transfection of the MLE-12 cells with sh-HOTAIR or sh-NC were also determined. The results showed that $m i R-30 a-5 p$ expression level was increased in the sh-HOTAIR group compared with that in the sh-NC group $(\mathrm{P}<0.01$; Fig. 2B). Subsequently, a DLR assay was performed to further verify the interaction between HOTAIR and $m i R-30 a-5 p$. The results showed that luciferase activity was lower in the HOTAIR WT + miR-30a-5p mimics group compared with that in the HOTAIR WT + miR-NC group $(\mathrm{P}<0.01$; Fig. 2C).

Overexpression of miR-30a-5p decreases the concentration of the inflammatory factors in the LPS-induced MLE-12 cells. The mRNA expression level of $m i R-30 a-5 p$ was significantly lower in the LPS group compared with that in the control group $(\mathrm{P}<0.01$; Fig. 3A). The transfection efficiency of the miR-30a-50p mimics, inhibitor and their respective NCs in the MLE-12 cells was determined using RT-qPCR. The results revealed that $m i R-30 a-5 p$ mRNA expression levels were downregulated by the miR-30a-5p inhibitor and upregulated by $m i R-30 a-5 p$ mimics $(\mathrm{P}<0.01$; Fig. $3 \mathrm{~B})$. After treatment with LPS, the results from a MTT assay and ELISA demonstrated that compared with that in the miR-NC group, cell viability 
A

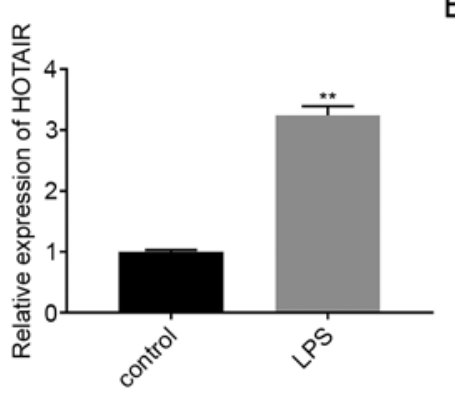

D

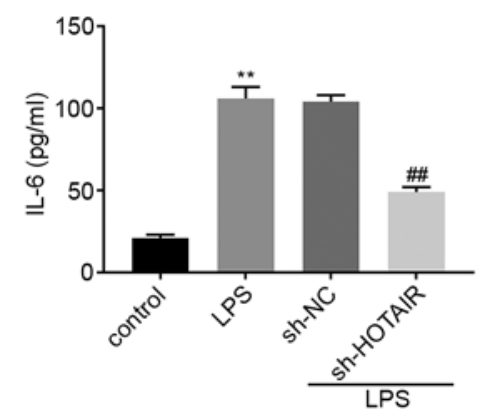

B

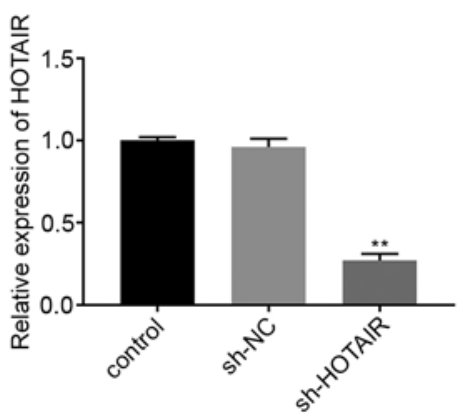

E

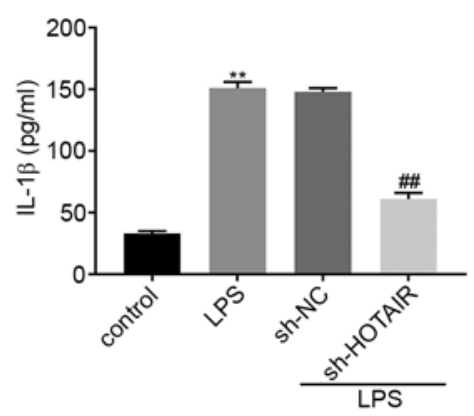

C

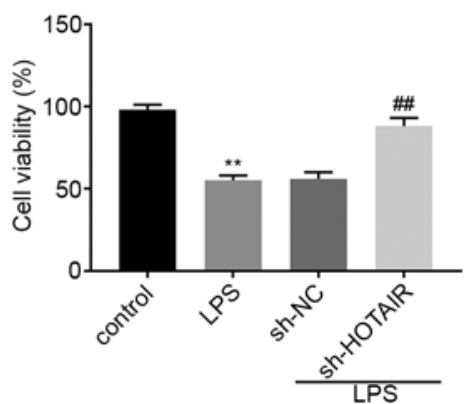

F

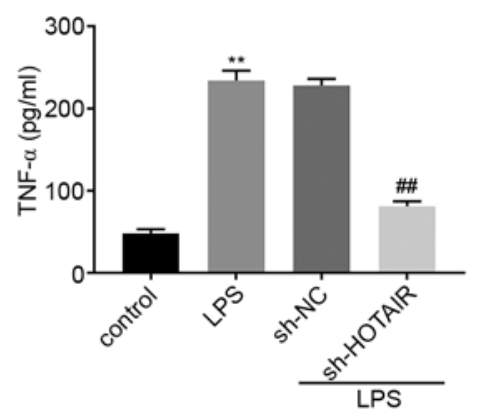

Figure 1. IncRNA HOTAIR knockdown decreases the concentration of inflammatory factors in LPS-induced MLE-12 cells. (A) The mRNA expression level of lncRNA HOTAIR in the MLE-12 cells was detected using RT-qPCR. ${ }^{* *} \mathrm{P}<0.01$ vs. control. (B) The mRNA expression of lncRNA HOTAIR following transfection with sh-HOTAIR/NC into the MLE-12 cells was detected using RT-qPCR. ${ }^{* *} \mathrm{P}<0.01$ vs. sh-NC. (C) The viability of the MLE-12 cells was measured using a MTT assay. The concentration of (D) IL-6, (E) IL-1 $\beta$ and (F) TNF- $\alpha$ following transfection with sh-HOTAIR/NC into the MLE-12 cells was measured using ELISA. ${ }^{* *} \mathrm{P}<0.01$ vs. control. ${ }^{\# /} \mathrm{P}<0.01$ vs. sh-NC. NC, negative control; lnc, long non-coding; LPS, lipopolysaccharide; TNF, tumor necrosis factor; RT-qPCR, reverse transcription-quantitative PCR; sh, short hairpin.

A

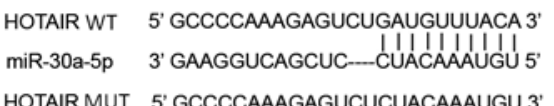
HOTAIRMUT 5 GCCCCAAAGAGUCUCUACAAAUGU
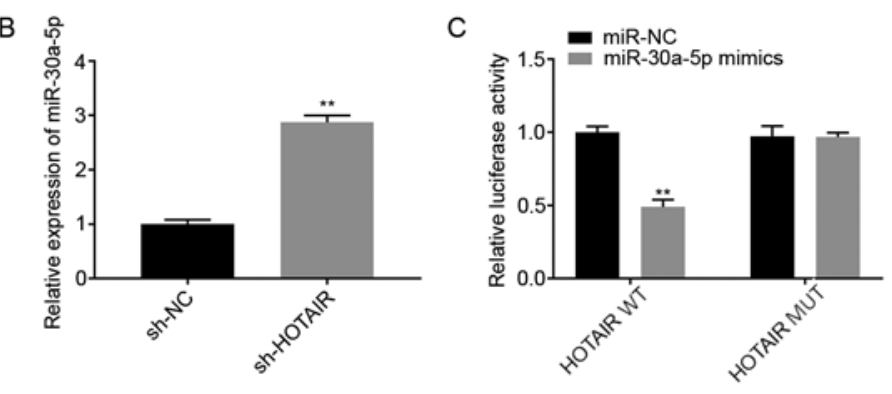

Figure 2. miR-30a-5p is the direct target of lncRNA HOTAIR. (A) The predicted complementary binding site between lncRNA HOTAIR and miR-30a-5p. (B) The mRNA expression level of miR-30a-5p following transfection with sh-HOTAIR/NC into the MLE-12 cells was detected using reverse transcriptionquantitative PCR. ${ }^{* *} \mathrm{P}<0.01$ vs. sh-NC. (C) The luciferase activity in the MLE-12 cells co-transfected with pGL3-HOTAIR WT/pGL3-HOTAIR MUT and miR-30a-5p mimics/NC was determined using a dual luciferase reporter assay. ${ }^{* *} \mathrm{P}<0.01 \mathrm{vs}$. miR-NC. NC, negative control; lnc, long non-coding; miR, microRNA, sh, short hairpin; WT, wild-type; MUT, mutant.

and the concentration of IL-6, IL-1 $\beta$ and TNF- $\alpha$ was increased and decreased, respectively, in the miR-30a-5p mimics group (P<0.01; Fig. 3C-F).

miR-30a-5p targets PDE7A. Using TargetScan database, the potential binding site between $m i R-30 a-5 p$ and PDE7A was predicted (Fig. 4A). A total of 1,570 targets were predicted. PDE7A was selected for further analysis as it has been associated with pulmonary inflammatory diseases, such as chronic obstructive pulmonary disease and asthma (24). Western blot analysis showed that the protein expression levels of PDE7A were decreased in the miR-30a-5p mimics group compared with that in the miR-NC group ( $\mathrm{P}<0.01$; Fig. 4B). DLR assays showed that luciferase activity was lower in the PDE7A
WT + miR-30a-5p mimics group compared with that in the PDE7A WT + miR-NC group $(\mathrm{P}<0.01$; Fig. $4 \mathrm{C})$.

IncRNA HOTAIR knockdown decreases inflammatory factor concentration in LPS-induced MLE-12 cells by regulating the miR-30a-5p/PDE7A axis. Western blot analysis was used to determine the protein expression levels of PDE7A in LPS-induced MLE-12 cells. The results showed that LPS treatment significantly increased PDE7A protein expression levels ( $\mathrm{P}<0.01$; Fig. 5A). pcDNA-PDE7A or pcDNA-NC were then transfected into the MLE-12 cells and the results from western blot analysis demonstrated that PDE7A overexpression significantly increased the protein expression levels of PDE7A (P<0.01; Fig. 5B). Subsequently, sh-NC, sh-HOTAIR, 

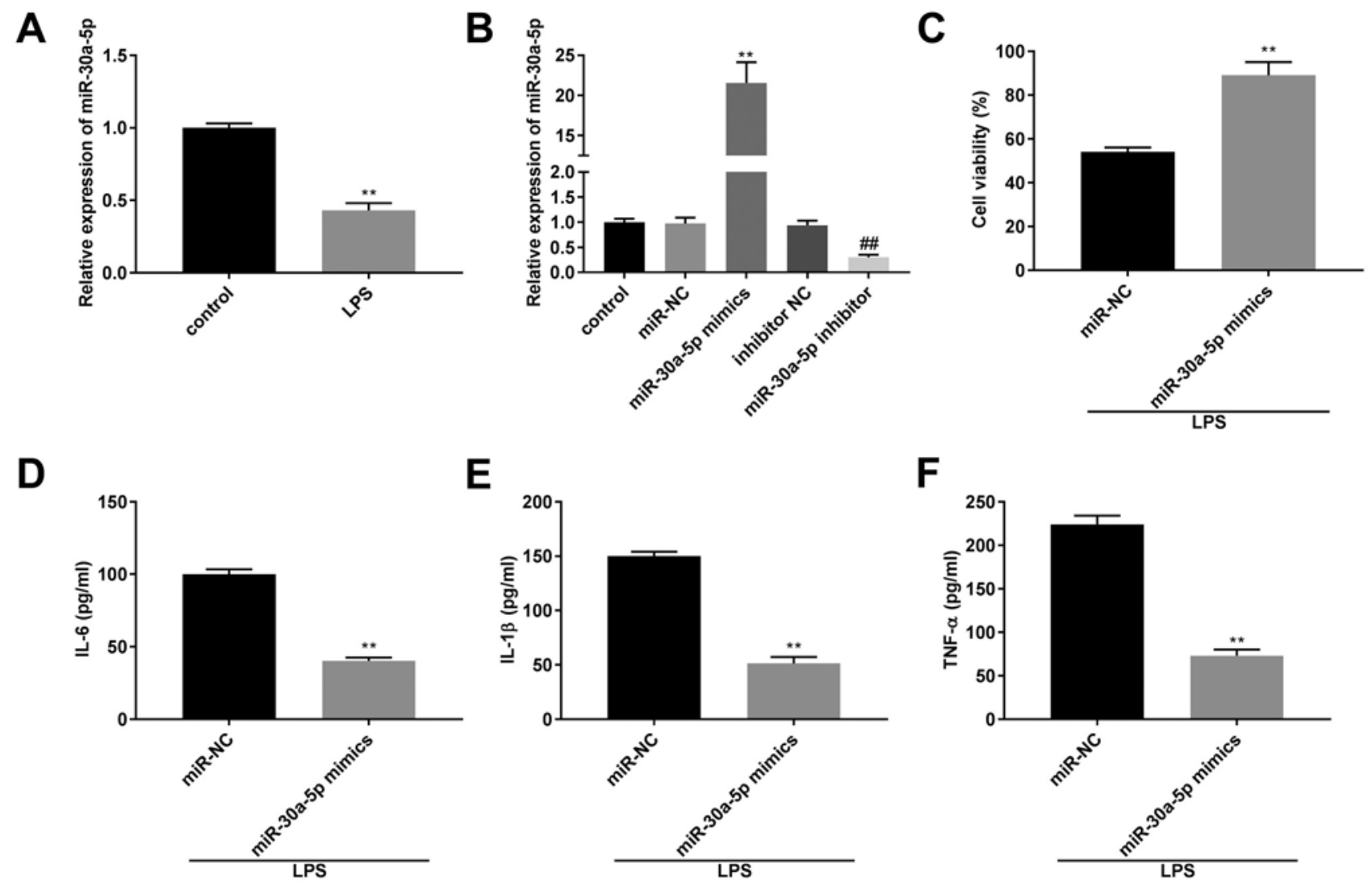

Figure 3. Overexpression of miR-30a-5p reduces the concentration of the inflammatory factors in LPS-induced MLE-12 cells. (A) The mRNA expression level of miR-30a-5p in the MLE-12 cells was detected using RT-qPCR. ${ }^{* *} \mathrm{P}<0.01$ vs. control. (B) The mRNA expression level of miR-30a-5p following transfection with miR-30a-5p mimics/inhibitor/NC into the MLE-12 cells was detected using RT-qPCR. (C) The viability of the MLE-12 cells was measured using a MTT assay. The concentration of (D) IL-6, (E) IL-1 $\beta$ and (F) TNF- $\alpha$ following transfection with miR-30a-5p mimics/NC into the MLE-12 cells was measured using ELISA. ${ }^{* *} \mathrm{P}<0.01$ vs. miR-NC. ${ }^{\# \#} \mathrm{P}<0.01$ vs. inhibitor NC. NC, negative control; miR, microRNA; LPS, lipopolysaccharide; TNF, tumor necrosis factor; RT-qPCR, reverse transcription-quantitative PCR.

A

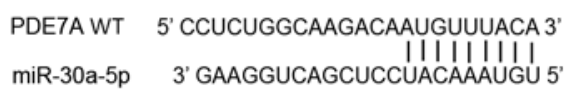
PDETAMUT 5 ' CCUCUGgCAagacauacAaAugu 3 '

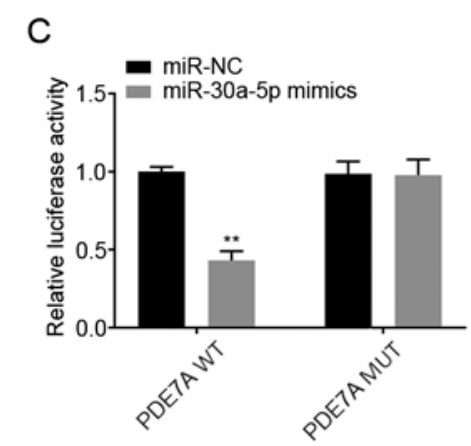

B

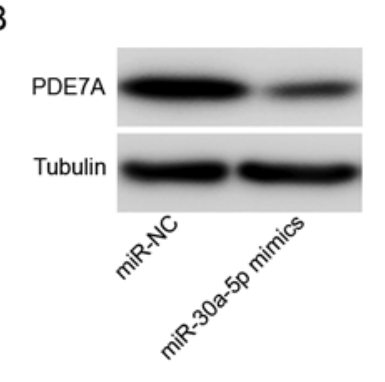

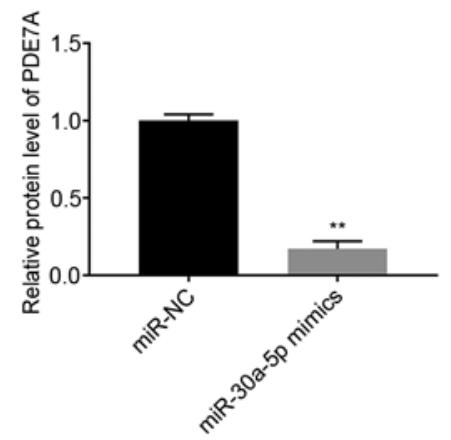

Figure 4. miR-30a-5p targets PDE7A. (A) The predicted complementary binding site between miR-30a-5p and PDE7A. (B) The protein expression level of PDE7A following transfection with miR-30a-5p mimics/NC into the MLE-12 cells was determined using western blot analysis. (C) The luciferase activity in the MLE-12 cells co-transfected with pGL3-PDE7A WT/pGL3-PDE7A MUT and miR-30a-5p mimics/NC was determined using a dual luciferase reporter assay. ${ }^{* *} \mathrm{P}<0.01$ vs. miR-NC. NC, negative control; miR, microRNA; WT, wild-type; MUT, mutant.

or sh-HOTAIR + miR-30a-5p inhibitor was transfected into the MLE-12 cells, then stimulated with LPS. The protein expression level of PDE7A was subsequently analyzed and the results indicated that HOTAIR knockdown decreased the 
A

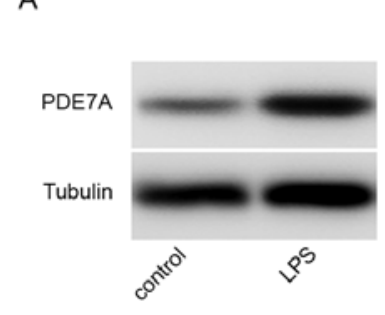

C

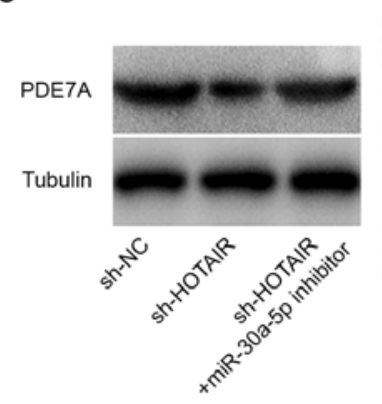

E

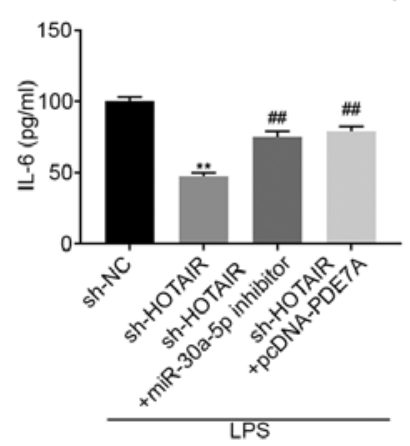

$\mathrm{F}$
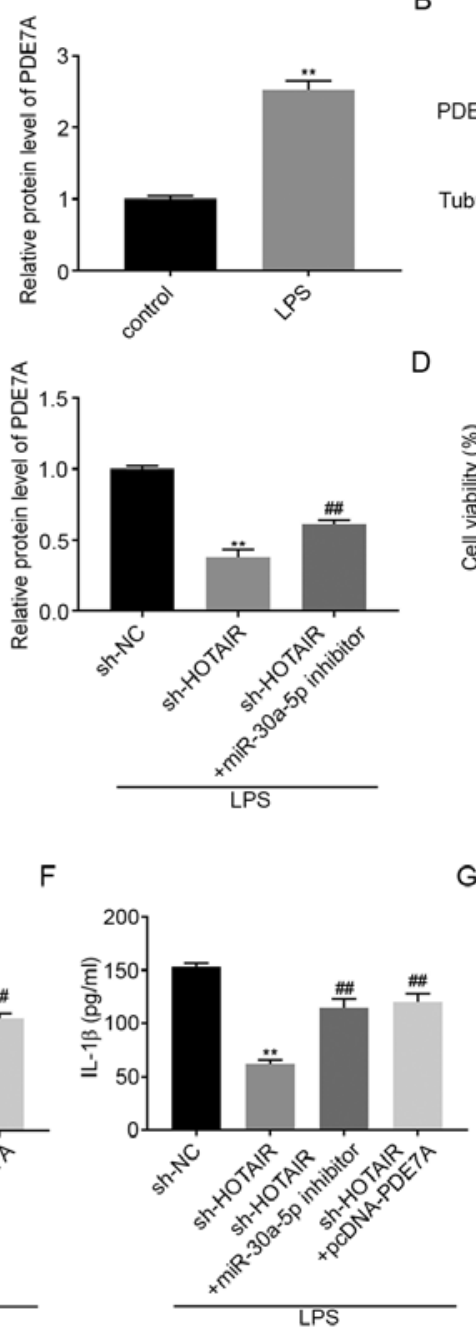

G
B
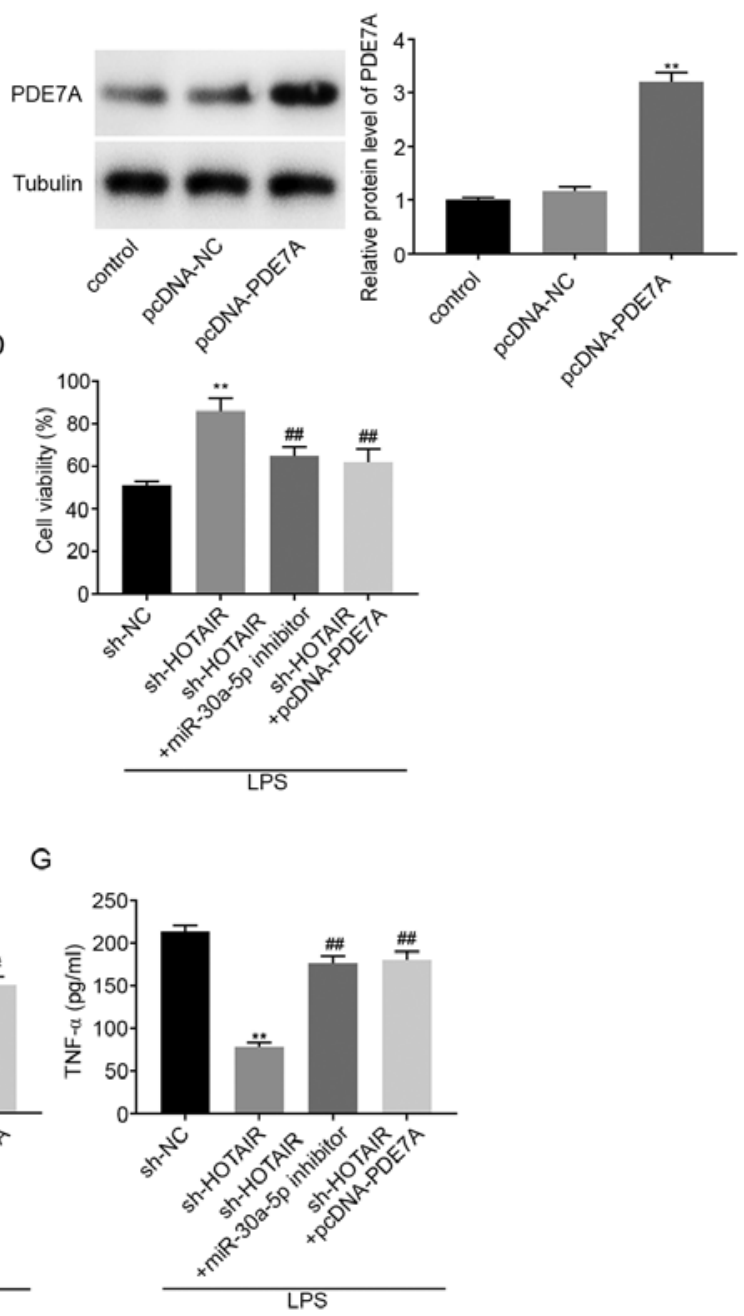

Figure 5. IncRNA HOTAIR knockdown decreases the concentration of the inflammatory factors in LPS-induced MLE-12 cells by regulating the miR-30a-5p/PDE7A axis. (A) The protein expression level of PDE7A in the MLE-12 cells was determined using a western blot assay. ${ }^{* *} \mathrm{P}<0.01$ vs. control. (B) The protein expression level of PDE7A following transfection with pcDNA-PDE7A/NC into the MLE-12 cells was determined using western blot analysis. ${ }^{* *} \mathrm{P}<0.01$ vs. pcDNA-NC. (C) The protein expression level of PDE7A following transfection with sh-HOTAIR + miR-30a-5p inhibitor/ sh-HOTAIR/NC into the MLE-12 cells was determined using western blot analysis. ${ }^{* *} \mathrm{P}<0.01 \mathrm{vs}$. sh-NC. ${ }^{\# \#} \mathrm{P}<0.01$ vs. sh-HOTAIR. (D) The viability of the MLE-12 cells was measured using a MTT assay. The level of (E) IL-6, (F) IL-1 $\beta$ and (G) TNF- $\alpha$ following transfection with sh-HOTAIR + miR-30a-5p inhibitor/sh-HOTAIR + pcDNA-PDE7A/sh-HOTAIR/NC into the MLE-12 cells was measured using ELISA. ${ }^{* *} \mathrm{P}<0.01$ vs. sh-NC. ${ }^{\# \#} \mathrm{P}<0.01$ vs. sh-HOTAIR NC, negative control; miR, microRNA; LPS, lipopolysaccharide; TNF, tumor necrosis factor; sh, short hairpin.

protein expression level of PDE7A, whereas downregulation of $m i R-30-5 p$ reversed the inhibitory effect of HOTAIR knockdown on PDE7A protein expression levels $(\mathrm{P}<0.01$; Fig. 5C). The results from the MTT assay showed that cell viability increased in the sh-HOTAIR group compared with that in the sh-NC group, whereas cell viability in both the sh-HOTAIR + miR-30a-5p inhibitor group and the sh HOTAIR + pcDNA-PDE7A group was partly reduced compared with that in the sh-HOTAIR group $(\mathrm{P}<0.01$; Fig. 5D). ELISA results showed that the concentrations of IL-6, IL-1 $\beta$, and TNF- $\alpha$ were all decreased by HOTAIR knockdown. However, downregulation of $m i R-30 a-5 p$ and upregulation of PDE7A both reversed the suppressive effect of HOTAIR knockdown on the concentration of the inflammatory factors $(\mathrm{P}<0.01$; Fig. 5E-G).

lncRNA HOTAIR knockdown attenuates LPS-induced $A R D S$ and the ARDS-related inflammatory response in vivo.
The mRNA expression levels of HOTAIR, miR-30a-5p, and $P D E 7 A$ in mouse lung tissues were determined using RT-qPCR. The results showed that, compared with that in the sham group, HOTAIR and PDE7A mRNA expression levels were elevated in the ARDS group, whereas $m i R-30 a-5 p$ mRNA expression levels were lower in the ARDS group $(\mathrm{P}<0.05$; Fig. 6A-C). Following an intravenous injection with sh-HOTAIR or sh-NC lentiviruses, the mRNA expression levels of HOTAIR and PDE7A were downregulated in the lung tissues of mice in the ARDS + sh-HOTAIR group compared with that in the ARDS group. However, miR-30a-5p mRNA expression levels were upregulated in the ARDS + sh-HOTAIR group compared with that in the ARDS group. As shown in Fig. 6D, the histopathological changes and injury scores of lung tissues were analyzed using H\&E staining. Inflammatory cell infiltration and interstitial oedema were more severe in the ARDS group compared with that in the sham group. HOTAIR knockdown markedly ameliorated these effects. The injury scores 
A

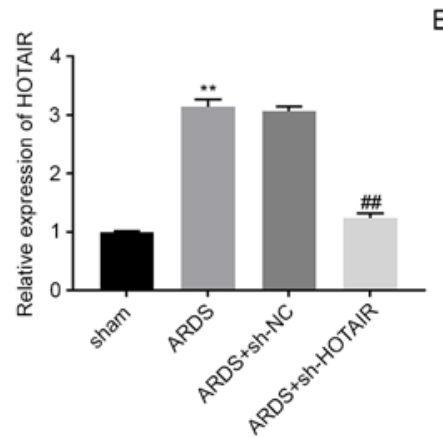

B

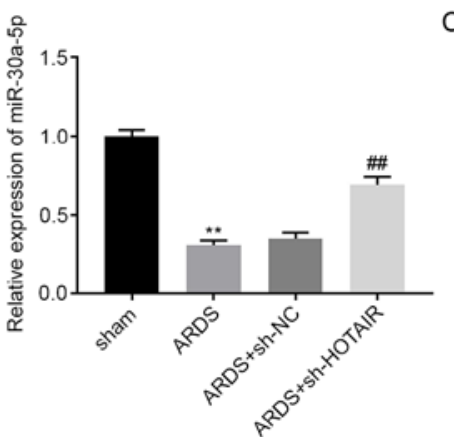

C

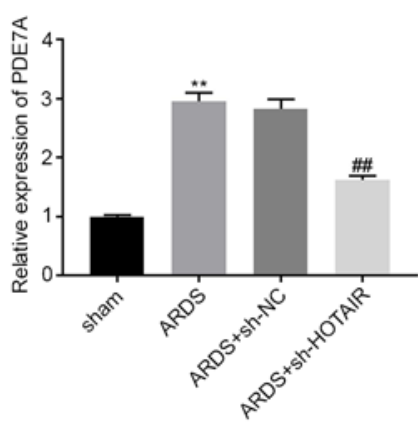

D
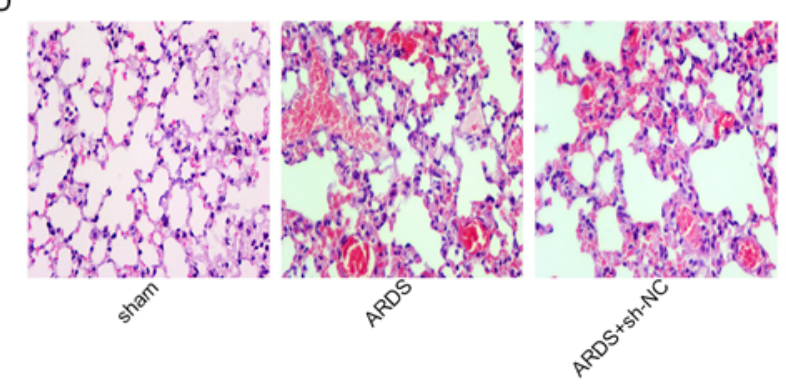

E

$\mathrm{F}$
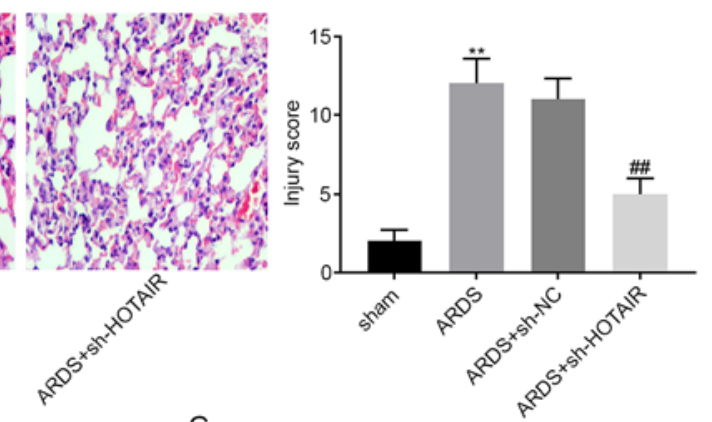

G
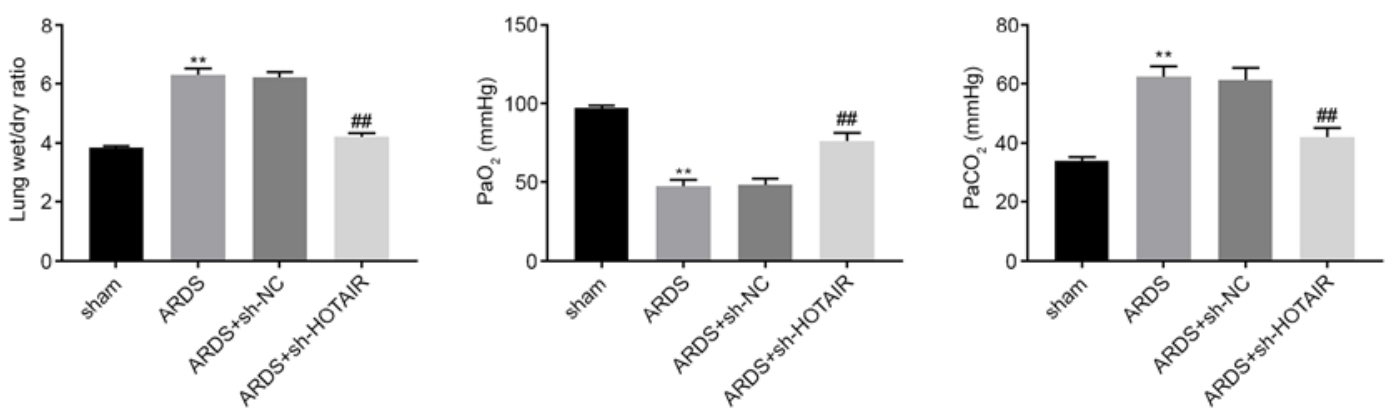

$\mathrm{H}$

I

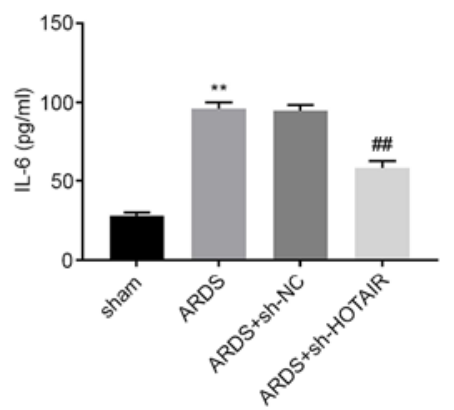

$\mathrm{J}$

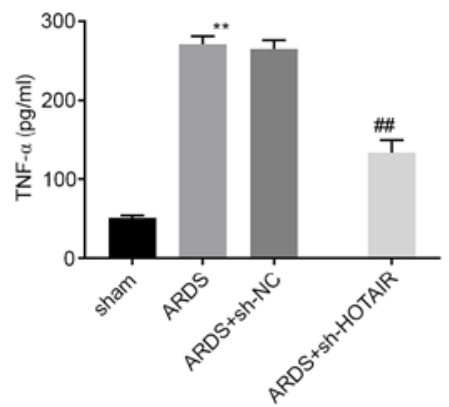

Figure 6. lncRNA HOTAIR knockdown attenuates LPS-induced ARDS and ARDS-related inflammatory response in vivo. The mRNA expression level of (A) HOTAIR, (B) miR-30a-5p and (C) PDE7A in the lung tissues of mice was detected using reverse transcription-quantitative PCR. (D) Histopathological change and injury score were detected using $\mathrm{H} \& \mathrm{E}$ staining. Magnification, $\mathrm{x} 200$. (E) Lung wet/dry weight ratio, (F) $\mathrm{PaO}_{2}$ and $(\mathrm{G}) \mathrm{PaCO}_{2}$ was measured using an automatic blood gas analyzer. The concentration of (H) IL-6, (I) IL-1 $\beta$ and (J) TNF- $\alpha$ in BALF of mice was measured using ELISA. * $P<0.01$ vs. sham. ${ }^{\# \# P} \mathrm{P}<0.01$ vs. ARDS. Pa, partial pressure; TNF, tumor necrosis factor; miR, microRNA; NC, negative control; sh, short hairpin; ARDS, acute respiratory distress syndrome.

were higher in the ARDS group compared with that in the sham group. HOTAIR knockdown significantly decreased the injury scores of the lung tissues $(\mathrm{P}<0.01$; Fig. $6 \mathrm{D})$. Lung function assays demonstrated that, compared with that in the sham group, $\mathrm{PaCO}_{2}$ levels and the lung wet/dry weight ratios were increased, whereas $\mathrm{PaO}_{2}$ levels were decreased in the ARDS group ( $\mathrm{P}<0.01$; Fig. 6E-G). HOTAIR knockdown decreased $\mathrm{PaCO}_{2}$ levels and lung wet/dry weight ratios, and increased $\mathrm{PaO}_{2}$ levels. ELISA results showed that IL-6, IL-1 $\beta$ and TNF- $\alpha$ concentration in the mouse BALF samples were all elevated in the ARDS group compared with that in the sham group $(\mathrm{P}<0.01$; Fig. 6H-J). However, HOTAIR knockdown significantly reversed the promoting effect of ARDS on inflammatory factor levels.

\section{Discussion}

As a severe form of ALI, the cost to treat ARDS is high, which severely affects the health and quality of life in patients with 
ARDS (25). Several lncRNAs have been associated with the progression of ALI and ARDS (7-9). Dai et al (7) found high protein expression levels of MALAT1 in the lung tissues from an LPS-induced rat ALI model. Wang et al (8) showed that THRIL expression was upregulated in lung tissues from patients with ARDS. Zhou et al (9) demonstrated that NEAT1 was highly expressed in the lung tissues of mice with LPS-induced ALI and in LPS-treated mouse alveolar epithelial cells. Consistent with the aforementioned results, the present study showed that HOTAIR mRNA expression levels were increased in the lung tissues from mice with LPS-induced ARDS and in LPS-treated MLE-12 cells. Therefore, the results from the present study suggested that HOTAIR may act as a pathogenic factor in ARDS.

Previous studies have reported that lncRNAs act as crucial regulators in hypoxic lung diseases and inflammatory epithelial injury $(26,27)$, by affecting processes, such as inflammation and interstitial oedema $(7,28,29)$. Dai et al $(7)$ have shown that MALAT1 inhibition led to a marked decrease in the concentration of IL- 6 , IL- $1 \beta$ and TNF- $\alpha$ in rat lung tissues. In addition, histopathological examination showed that MALAT1 inhibition distinctly attenuated lung tissue injury. Jiang et al (29) showed that the $\mathrm{PaO}_{2} / \mathrm{FiO}_{2}$ ratio was decreased and the lung wet/dry weight ratio was increased in ARDS. Kcnq1ot1 knockdown in mice was shown to reverse the suppressive effect of LPS on the $\mathrm{PaO}_{2} / \mathrm{FiO}_{2}$ ratio and the positive effect of LPS on the lung wet/dry weight ratio (29). Consistent with the aforementioned results, in the present study, it was found that injury scores, lung wet/dry weight ratios, $\mathrm{PaCO}_{2}$ levels and the concentration of IL-6, IL-1 $\beta$ and TNF- $\alpha$ were increased, and $\mathrm{PaO}_{2}$ levels were decreased in a mouse model of ARDS. HOTAIR knockdown using a sh HOTAIR lentivirus injection reversed the positive effect of LPS on injury scores, lung wet/dry weight ratios, $\mathrm{PaCO}_{2}$ levels, and the concentration of IL- 6 , IL- $1 \beta$ and TNF- $\alpha$, and the negative effect of $\mathrm{LPS}$ on $\mathrm{PaO}_{2}$ levels. These results suggested that HOTAIR knockdown attenuated the LPS-induced inflammatory response and LPS-induced ALI. To further verify this hypothesis, further experiments were performed using the MLE-12 cells and it was found that sh-HOTAIR reversed the negative effect of LPS on cell viability and the positive effect of LPS on the concentration of IL-6, IL- $1 \beta$ and TNF- $\alpha$. The results from the present study are consistent with those of other studies (10-12), suggesting that HOTAIR knockdown attenuated the LPS-induced inflammatory response and LPS-induced ALI in vivo and in vitro.

Increasing evidence has indicated that miRNA expression levels were downregulated in LPS-induced rat models of ALI or LPS-treated cells. For example, miR-497a-5p (30) and $m i R-381-3 p$ (29) expression levels were downregulated in an LPS-induced mouse model of ALI, while miR-424 (14) expression levels were downregulated in alveolar epithelial cells, and $m i R-146 b$ (15) expression levels were downregulated in murine lung alveolar macrophages. Similarly, in the present study it was found that $m i R-30 a-5 p$ expression levels were downregulated in both an LPS-induced mouse model of ARDS and in LPS-treated MLE-12 cells. In addition, previous studies have shown that miRNAs have a role in modulating pulmonary inflammation $(13,14,30)$. Cheng et al (14) showed that upregulation of $m i R-424$ suppressed the secretion of IL-6 and IL-8 from
LPS-treated alveolar epithelial cells. Guo et al (30) showed that $m i R-497 a-5 p$ overexpression decreased the concentration of IL-6, IL- $1 \beta$ and TNF- $\alpha$ in LPS-treated RAW264.7 cells. Li et al (13) reported that high expression levels of $m i R-150$ inhibited the secretion of IL-6, IL- $1 \beta$ and TNF- $\alpha$ from LPS-treated human pulmonary epithelial cells. In the present study, it was found that $m i R-30 a-5 p$ upregulation suppressed the secretion of IL- 6, IL- $1 \beta$ and TNF- $\alpha$ from LPS-treated MLE-12 cells. These results suggested that miR-30a-5p may be a suppressor of the inflammatory response. Consistent with these results, $m i R-30 a-5 p$ inhibition has been shown to promote the release of IL- 6 , IL- $1 \beta$ and TNF- $\alpha$ from PC- 12 cells (31). In addition, $m i R-30 a-5 p$ was shown to be the target of HOTAIR in the present study. Downregulation of $m i R-30 a-5 p$ reversed the positive effect of sh-HOTAIR on MLE-12 cell viability and the inhibitory effect of sh-HOTAIR on inflammatory factor release from MLE-12 cells. This suggested that HOTAIR knockdown alleviated the LPS-induced inflammatory response by modulating $m i R-30 a-5 p$.

Enzymes within the phosphodiesterase (PDE) family are found in most proinflammatory and immune cells (24). As a member of the PDE family, PDE7A has been associated with the inflammatory response $(24,32$ 34). Goto et al (32) reported that PDE7A downregulation ameliorated concanavalin A-induced hepatitis in mice by suppressing the concentration of IL- 4 and TNF- $\alpha$. Kadoshima-Yamaoka et al (33) showed that ASB16165, a PDE7A inhibitor, reduced cutaneous TNF- $\alpha$ concentration in 12-o-tetradecanoylphorbol-13-acetate-induced skin inflammation in mice. Yamamoto et al (34) demonstrated that YM-393059, an inhibitor of PDE7A, inhibited LPS-induced TNF- $\alpha$ production in mice. In the present study, it was found that PDE7A protein expression levels were upregulated in LPS-treated MLE-12 cells and PDE7A overexpression accelerated inflammatory factor release from the MLE-12 cells. These results suggested that PDE7A was associated with promoting the secretion of the inflammatory factors, whereas inhibiting PDE7A may be beneficial in preventing inflammation in ARDS. In addition, PDE7A overexpression reversed the positive effect of sh-HOTAIR on cell viability and the inhibitory effect of sh-HOTAIR on inflammatory factor release from the MLE-12 cells. This suggested that HOTAIR knockdown attenuated the LPS-induced inflammatory response by regulating PDE7A. At the same time, PDE7A was the target gene of $m i R-30 a-5 p$. We further hypothesized that knockdown of HOTAIR ameliorated the LPS-induced inflammatory response by regulating the $m i R-30 a-5 p / P D E 7 A$ axis.

Taken together, the results of the current study showed that HOTAIR knockdown ameliorated the LPS-induced inflammatory response and ARDS in vivo and reduced LPS-induced inflammatory factor production by regulating the miR-30a-5p/PDE7A axis in vitro. However, there were also some limitations. First, the detailed mechanism involving HOTAIR, miR-30a-5p and PDE7A was not confirmed in vivo. Second, the detailed mechanism of this axis and the signaling pathways involved remain unclear. Further experiments will be performed to elucidate these in the future. In conclusion, these findings may contribute to the development of a new strategy for treating ARDS. 


\section{Acknowledgements}

Not applicable.

\section{Funding}

No funding was received.

\section{Availability of data and materials}

All data generated or analyzed during this study are included in this published article.

\section{Authors' contributions}

XM conceived and designed the present study. HW and SS performed the experiments, analyzed the data and drafted the article. XM revised the article critically for important intellectual content. XM, HW and SS confirm the authenticity of all the raw data. All authors have read and approved the final manuscript.

\section{Ethics approval and consent to participate}

All experimental procedures were conducted in agreement with the principles approved by the ethical committee of Yantai Yuhuangding hospital (Yantai, China; approval no. 2019017).

\section{Patient consent for publication}

Not applicable.

\section{Competing interests}

The authors declare that they have no competing interests.

\section{References}

1. Patel VJ, Roy SB, Mehta HJ, Joo M and Sadikot RT: Alternative and Natural therapies for acute lung injury and acute respiratory distress syndrome. Biomed Res Int 2018: 2476824, 2018.

2. Bellani G, Laffey JG, Pham T, Fan E, Brochard L, Esteban A, Gattinoni L, van Haren F, Larsson A, McAuley DF, et al: Epidemiology, patterns of care, and mortality for patients with acute respiratory distress syndrome in intensive care units in 50 countries. JAMA 315: 788-800, 2016.

3. Villar J, Blanco J and Kacmarek RM: Current incidence and outcome of the acute respiratory distress syndrome. Curr Opin Crit Care 22: 1-6, 2016

4. Tsai CL, Lin YC, Wang HM and Chou TC: Baicalein, an active component of Scutellaria baicalensis, protects against lipopolysaccharide-induced acute lung injury in rats. J Ethnopharmacol 153: 197-206, 2014.

5. Opitz B, Van Laak V, Eitel J and Suttorp N: Innate immune recognition in infectious and noninfectious diseases of the lung. Am J Respir Crit Care Med 181: 1294-1309, 2010.

6. Han S and Mallampalli RK: The acute respiratory distress syndrome: From mechanism to translation. J Immunol 194: 855-860, 2015.

7. Dai L, Zhang G, Cheng Z, Wang X, Jia L, Jing X, Wang H, Zhang R, Liu M, Jiang T, et al: Knockdown of LncRNA MALAT1 contributes to the suppression of inflammatory responses by up-regulating miR-146a in LPS-induced acute lung injury. Connect Tissue Res 59: 581-592, 2018.

8. Wang Y, Fu X, Yu B and Ai F: Long non-coding RNA THRIL predicts increased acute respiratory distress syndrome risk and positively correlates with disease severity, inflammation, and mortality in sepsis patients. J Clin Lab Anal 33: e22882, 2019.
9. Zhou H, Wang X and Zhang B: Depression of lncRNA NEAT1 Antagonizes LPS-evoked acute injury and inflammatory response in alveolar epithelial cells via HMGB1-RAGE signaling. Mediators Inflamm 2020: 8019467, 2020.

10. Duan G, Song S and Niu S: WITHDRAWN: Long non-coding RNA HOTAIR promotes LPS-induced inflammatory injury by down-regulation of microRNA-124 in murine chondrogenic ATDC5 cells. Life Sci: July 20, 2018 (Epub ahead of print).

11. Obaid M, Udden SMN, Deb P, Shihabeddin N, Zaki MH and Mandal SS: LncRNA HOTAIR regulates lipopolysaccharide-induced cytokine expression and inflammatory response in macrophages. Sci Rep 8: 15670, 2018.

12. Wu H, Liu J, Li W, Liu G and Li Z: LncRNA-HOTAIR promotes TNF- $\alpha$ production in cardiomyocytes of LPS-induced sepsis mice by activating NF- $\kappa$ B pathway. Biochem Biophys Res Commun 471: 240-246, 2016.

13. Li P, Yao Y, Ma Y and Chen Y: MiR-150 attenuates LPS-induced acute lung injury via targeting AKT3. Int Immunopharmacol 75: 105794, 2019.

14. Cheng D, Zhu C, Liang Y, Xing Y and Shi C: MiR-424 overexpression protects alveolar epithelial cells from LPS-induced apoptosis and inflammation by targeting FGF2 via the NF- $\kappa \mathrm{B}$ pathway. Life Sci 242: 117213, 2020.

15. He R, Li Y, Zhou L, Su X, Pan P and Hu C: miR-146b overexpression ameliorates lipopolysaccharide-induced acute lung injury in vivo and in vitro. J Cell Biochem 120: 2929-2939, 2019.

16. Zhou T and Chen YL: The functional mechanisms of miR-30b-5p in acute lung injury in children. Med Sci Monit 25: 40-51, 2019.

17. Zhang Y, Ai H, Fan X, Chen S, Wang Y and Liu L: Knockdown of long non-coding RNA HOTAIR reverses cisplatin resistance of ovarian cancer cells through inhibiting miR-138-5p-regulated EZH2 and SIRT1. Biol Res 53: 18, 2020.

18. Wei Z, Chen L, Meng L, Han W, Huang L and Xu A: LncRNA HOTAIR promotes the growth and metastasis of gastric cancer by sponging miR-1277-5p and upregulating COL5A1. Gastric Cancer 23: 1018-1032, 2020.

19. Zhang S, Wang B, Xiao H, Dong J, Li Y, Zhu C, Jin Y, Li H, Cui M and Fan S: LncRNA HOTAIR enhances breast cancer radioresistance through facilitating HSPA1A expression via sequestering miR-449b-5p. Thorac Cancer 11: 1801-1816, 2020.

20. Wang Y, Gong G, Xu J, Zhang Y, Wu S and Wang S: Long noncoding RNA HOTAIR promotes breast cancer development by targeting ZEB1 via sponging miR-601. Cancer Cell Int 20: 320, 2020.

21. Zhang C, Xu L, Deng G, Ding Y, Bi K, Jin H, Shu J, Yang J, Deng $\mathrm{H}$, Wang $\mathrm{Z}$ and Wang Y: Exosomal HOTAIR promotes proliferation, migration and invasion of lung cancer by sponging miR-203. Sci China Life Sci 63: 1265-1268, 2020.

22. Livak KJ and Schmittgen TD: Analysis of relative gene expression data using real-time quantitative PCR and the 2(-Delta Delta C(T)) method. Methods 25: 402-408, 2001.

23. Guo Z, Li Q, Han Y, Liang Y, Xu Z and Ren T: Prevention of LPS-induced acute lung injury in mice by progranulin. Mediators Inflamm 2012: 540794, 2012

24. Smith SJ, Brookes-Fazakerley S, Donnelly LE, Barnes PJ, Barnette MS and Giembycz MA: Ubiquitous expression of phosphodiesterase 7A in human proinflammatory and immune cells. Am J Physiol Lung Cell Mol Physiol 284: L279-L289, 2003.

25. Petrucci $\mathrm{N}$ and De Feo C: Lung protective ventilation strategy for the acute respiratory distress syndrome. Cochrane Database Syst Rev 2013: CD003844, 2007.

26. Sun H, Chen J, Qian W, Kang J, Wang J, Jiang L, Qiao L, Chen $\mathrm{W}$ and Zhang J: Integrated long non-coding RNA analyses identify novel regulators of epithelial-mesenchymal transition in the mouse model of pulmonary fibrosis. J Cell Mol Med 20: 1234-1246, 2016.

27. Liang H, Gu Y, Li T, Zhang Y, Huangfu L, Hu M,Zhao D, Chen Y, Liu S, Dong Y, et al: Integrated analyses identify the involvement of microRNA-26a in epithelial-mesenchymal transition during idiopathic pulmonary fibrosis. Cell Death Dis 5: e1238, 2014.

28. Dunkel B: Acute lung injury and acute respiratory distress syndrome in foals. Equine Vet J 5: 127-133, 2006.

29. Jiang X, Yu M, Zhu T, Lou L, Chen X, Li Q, Wei D and Sun R: Kcnq1ot1/miR-381-3p/ETS2 axis regulates inflammation in mouse models of acute respiratory distress syndrome. Mol Ther Nucleic Acids 19: 179-189, 2020.

30. Guo S, Chen Y, Liu J, Yang J, Yang C, Zhang T, Jiang K, Wu Z, Shaukat A and Deng G: miR-497a-5p attenuates lipopolysaccharide-induced inflammatory injury by targeting IRAK2. J Cell Physiol 234: 22874-22883, 2019. 
31. Zhu S, Zhou Z, Li Z, Shao J, Jiao G, Huang YE and Lin Y: Suppression of LINC00707 alleviates lipopolysaccharide-induced inflammation and apoptosis in PC-12 cells by regulated miR-30a-5p/Neurod 1. Biosci Biotechnol Biochem 83: 2049-2056, 2019.

32. Goto M, Tanaka Y, Murakawa M, Kadoshima-Yamaoka K, Inoue H, Murafuji H, Nagahira A, Kanki S, Hayashi Y, Nagahira K, et al: Inhibition of phosphodiesterase 7A ameliorates Concanavalin A-induced hepatitis in mice. Int Immunopharmacol 9: 1347-1351, 2009.
33. Kadoshima-Yamaoka K, Goto M, Murakawa M, Yoshioka R, Tanaka Y, Inoue H, Murafuji H, Kanki S, Hayashi Y, Nagahira K, et al: ASB16165, a phosphodiesterase 7A inhibitor, reduces cutaneous TNF-alpha level and ameliorates skin edema in phorbol ester 12-O-tetradecanoylphorbol-13-acetate-induced skin inflammation model in mice. Eur J Pharmacol 613: 163-166, 2009.

34. Yamamoto S, Sugahara S, Naito R, Ichikawa A, Ikeda K, Yamada T and Shimizu Y: The effects of a novel phosphodiesterase 7A and -4 dual inhibitor, YM-393059, on T-cell-related cytokine production in vitro and in vivo. Eur J Pharmacol 541: 106-114, 2006. 